\title{
La prevención del suicidio juvenil: entre la enunciación y la acción
}

\author{
Elsa Viviana Barrón \\ Universidad de Buenos Aires
}

\author{
Claudia Sandra Krmpotic \\ Centro Argentino de Etnología Americana, CONICET
}

\section{La prevención del suicidio juvenil: entre la enunciación y la acción}

Resumen: El artículo expone las estrategias argumentativas que explican al suicidio juvenil, sus características y las formas de prevención posibles a partir de la recopilación, lectura y análisis de contenido transversal de publicaciones de cuatro grupos: profesionales de la salud, sociólogos, filósofos existencialistas y teólogos que escribieron sobre la temática entre 1990 y 2011 . Se analiza la relevancia de los factores de riesgo como matriz explicativa y cuestiona su eficacia para operacionalizar servicios socio-sanitarios oportunos y de calidad.

Palabras clave: Suicidio juvenil. Factores de riesgo. Prevención. Servicios socio-sanitarios.

\section{A prevenção do suicídio juvenil: entre a enunciação e a ação.}

Resumo: O artigo apresenta as estratégias argumentativas que explicam o suicídio de jovens, suas características e possíveis formas de prevenção a partir da coleção, leitura e análise de conteúdo transversal de publicações de quatro grupos: profissionais da saúde, sociólogos, filósofos existencialistas e teólogos que escreveram sobre o tema entre 1990 e 2011. Analisa-se a relevância dos fatores de risco como matriz explicativa e questiona a sua eficácia para operacionalizar os serviços sociosanitarios oportunos e de qualidade.

Palavras-chave: Suicídio juvenil. Fatores de risco. Prevenção. Serviços Sociosanitarios.

\section{Prevention of Youth Suicide: between statement and action}

Abstract: This article presents the argumentative strategies that explain juvenile suicide, its characteristics and possible forms of prevention based on the compilation, reading and analysis of the transversal content of publications from four groups: healthcare professionals, sociologists, existential philosophers and theologians who have written about the theme from 1990 to 2011. It analyzes the relevance of the risk factors as an explanatory matrix and questions the effectiveness of this matrix for operationalizing timely and quality social and healthcare services.

Keywords: Youth suicide. Risk factors. Prevention. Social and healthcare services. 


\section{Introducción}

En la Argentina contemporánea el suicidio juvenil se ha convertido en un fenómeno creciente, que contraría las expectativas que la sociedad y el Estado construyen en torno a los jóvenes a través de los sistemas de incentivos. Por lo tanto, es objetivo de esta contribución recuperar los diferentes abordajes disciplinares que han construido explicaciones acerca del suicidio, a fin de identificar el entramado de creencias que sostienen que el suicidio puede evitarse y que la prevención es la única intervención posible. Para operacionalizar esta convicción, un servicio socio-sanitario oportuno y de calidad en la prevención del suicidio es definitivamente necesario en la comunidad. Si bien en su problematización se identifican tres nociones-clave, factores de riesgo, enfermedad mental y muerte, focalizaremos en los primeros dado que son los que estructuran la lógica preventiva.

Desde una lectura regionalmente localizada con foco en la realidad Argentina, consideramos el corpus de materiales recogidos en la perspectiva de las creencias, es decir, de las formas en que esos discursos modelan las certezas. Las creencias expresan además de un conjunto de conceptos, el posicionamiento de los autores respecto a un estado de cosas cuyo valor de verdad se sostiene a partir de una adhesión personal o interpersonal. Ciertos enunciados se aceptan como ciertos, por medio de operaciones subjetivas de aserción y de una retórica que procura convencer de dichas certezas. Trabajar en el plano de las creencias nos posibilita trascender las fronteras disciplinarias así como identificar puntos de intersección entre retórica y ciencia. Al igual que otras prácticas, la ciencia busca proveer de argumentos convincentes hasta forzar su legitimidad y reclamar así la autoridad para establecer definiciones y líneas de acción. Sea por medio del lenguaje o por medio de la lógica, en cualquier caso procuro persuadir, es decir, presentar con fuerza una argumentación sustentada en un uso científico pero también literario del lenguaje. En este sentido, y con el objeto de analizar los patrones argumentativos de algunas de estas creencias, en particular aquellas asociadas a los factores sociales y desencadenantes que llevan al suicidio juvenil, aplicamos la técnica de análisis de contenido sobre un corpus de publicaciones editadas entre 1990 y 2011 que reflejan la realidad argentina y americana, seleccionado a partir de un muestreo teórico.

Nos proponemos demostrar cómo una narrativa basada en factores de riesgo, junto a la ambigüedad interpretativa que algunos presentan y las resistencias institucionales para incorporar registros que ordenen las evidencias, ponen en duda el pasaje del análisis a la acción, lo que constituye una deuda que enfrentan los servicios sociales y sanitarios cuando se trata de reaccionar frente a la muerte joven. Para las profesiones de cuidado en general, y para el Trabajador Social en particular, el suicidio juvenil - sea en la ideación, el intento o la consumación - presenta nuevas exigencias en materia de diagnóstico social fundado, como respecto del reconocimiento de la interfaz socio-legal presente en estas situaciones que atañen no sólo al protagonista, sino a sus cuidadores como al entorno socio-familiar más amplio.

\section{Fundamentos}

El suicidio juvenil se ha convertido en un problema que afecta de manera constante a la población. En la Argentina, las tasas de muerte por suicidios aumentaron de 1,5 cada 100.000 habitantes en 1991 al 6,1 en el año 2000 (SERFATY, 2005). Analizando los datos oficiales, entre 2000 y 2007 fallecieron 44.829 jóvenes, con un promedio de 5.603 por año y unos 15 por día, de los cuales 10 fueron por causas evitables, mientras entre 2 y 3 por suicidio (BARRÓN, 2015). En América latina se registran reportes similares con tasas de mortalidad que llegan a ser 180\% más altas que las de países desarrollados (YUNES y ZUBAREW, 1999).

Las limitaciones en su difusión y el debate público por la presunción del efecto contagio invisibiliza la cuestión e inhibe procesos de toma en cuenta y responsabilidad social, quedando circunscripto a un problema individual que queda sumergido en la memoria familiar. Efectivamente, frente al suicidio consumado no hay nada que hacer salvo constreñir el efecto imitación y el impacto negativo en el entorno del joven fallecido. Ello se debe, en parte, al hecho que la prevención se torna en una creencia fundante: el suicidio puede evitarse, prevenirse, al igual que cualquier muerte. En ningún caso la muerte es la solución para los problemas de la vida. Hay consenso en la idea que el joven no debe decidir terminar con su vida. Si lo hace es que algo definitivamente no está bien.

Una lectura del estado del arte da cuenta de un núcleo básico de creencias recurrentes: i) el suicidio es un proceso que se inicia con una ideación, la muerte es premeditada, deliberada; ii) el suicidio es un acto que rompe con la racionalidad. En el origen del suicidio hay una decisión de poner fin a algo que difícilmente es la propia existencia del sujeto que muere; iii) los factores que llevan al suicidio se dividen en dos grandes grupos: predisponentes y desencadenantes. Estos últimos son siempre sociales. Por la profundidad de su impacto precipitan el pasaje al acto del suicidio, sin ser completamente responsables del suicidio. No obstante, se cree que sin la presencia de elementos predisponentes, aun con los mismos episodios desencadenantes, no se daría el suicidio consumado; iv) 
la familia tiene un rol protagónico en su desencadenamiento (familias inestables, abandónicas, violentas, extremadamente rígidas, ausentes; familias que no cuidan); y v) el ámbito educativo formal como la pertenencia a otros espacios de socialización informal, son efectivos en la prevención del suicidio.

En suma, lo social y sus problemas vitales se presentan a través de categorías de tan vasto alcance que podrían ser explicativas de un sinnúmero de problemas sociales: fracaso escolar, desempleo, desigualdades, familias disgregadas y escasas redes de pertenencia social, resumen los factores que desencadenan una decisión tan dramática como el suicidio. Asimismo, encontramos en las argumentaciones científicas la mención de aspectos espirituales de las personas, recurriendo a términos también presentes en un discurso espiritualista/religioso: culpa, dolor, falta de paz y de perdón, rechazo y auto-rechazo son nociones ampliamente citadas con variaciones sea que se trate del discurso científico o el religioso. Las diversas interpretaciones reflejan intersecciones argumentativas, lo que obedece, sin dudas, a la sacralización del tema de la muerte, pero también a la interacción de las ciencias con la religión cuando se llama la atención en torno a los límites del proceso de secularización del saber científico en la continuidad de formas explicativas de base teológica. Más aún, si analizamos las narrativas de sufrientes en el pensamiento en la vida cotidiana, las fronteras entre lo secular y lo religioso son imperceptibles, combinando ambos recursos en las interpretaciones de la propia vida y sus pesares.

\section{Metodología}

La búsqueda de las creencias y sus fundamentos se desarrolló a partir de la recopilación, lectura y análisis de contenido de materiales publicados provenientes de cuatro grupos: los profesionales de la salud, los sociólogos, los filósofos existencialistas y teólogos. Tomando como caso para este último grupo a los bautistas, representativos de religión y cura, sobre el que se realizó además una acabada etnografía ${ }^{1}$ (PINUELL RAIGADA y GAITÁN MOYA, 1995; ROBSON, 2002; SHUTZ, 1972). El corpus de documentos escritos se conformó a partir de la selección de materiales publicados (en soporte electrónico y papel) que presentaban argumentaciones sobre el suicidio en general y el suicidio juvenil en particular, como así también referidas a problemas asociados como las enfermedades mentales y adicciones, la mortalidad juvenil, la violencia relacionada con adolescentes y jóvenes, la masculinidad y la salud pública. La muestra incluyó artículos, tesis doctorales y capítulos de libros producidos entre 1990 y 2011, que fueron relevados en bibliotecas y bases de datos on-line (REDALYC, SCIELO, UNAM, ALAP, WHO, OPS), así como en librerías y bibliotecas de la Ciudad de Buenos Aires. La selección alcanzó un número de 68 materiales y 3712 páginas que dan cuenta de la realidad nacional y americana, procurando ver reflejadas en ellos las creencias que circulan localmente. Estas publicaciones a su vez refieren a 2467 obras que tienen el origen que se detalla a continuación:

\section{Cuadro 1: Origen de las referencias más utilizadas en el corpus de documentos escritos analizados}

\begin{tabular}{|c|c|c|}
\hline Origen de las obras de referencia & Frecuencia & Porcentaje \% \\
\hline EE UU & 655 & 26,55 \\
\hline OMS/OPS & 130 & 5,27 \\
\hline Canadá & 4 & 0,16 \\
\hline Europa (sin España) & 340 & 13,8 \\
\hline España & 285 & 11,55 \\
\hline América Latina & 863 & 34,98 \\
\hline Argentina & 163 & 6,61 \\
\hline Australia & 2 & 0,08 \\
\hline CEPAL & 24 & 0,96 \\
\hline UNICEF & 1 & 0,04 \\
\hline TOTAL & 2467 & 100 \\
\hline
\end{tabular}

Fuente: Elaboración propia, resultado del meta-análisis. 
Para seleccionar las publicaciones se empleó el criterio de muestreo teórico, teniendo en cuenta la información que cada material aporta a la comprensión del suicidio juvenil, desde las diferentes disciplinas investigadas. El muestreo se realizó a partir de su contenido como así también considerando la frecuencia con la que eran citados en otros materiales. Para el trabajo con los textos, se trabajó con la técnica de análisis de contenido, que se orientó hacia la búsqueda y comparación de los patrones de argumentación sobre el suicidio en los cuatro grupos mencionados, hasta el punto de saturación a los fines de evitar repeticiones innecesarias. A partir de un análisis de contenido transversal seleccionamos muestras textuales del corpus en las que se expresan las creencias acerca del suicidio, para luego analizarlas teniendo en cuenta sus contextos de producción. Se procedió luego a una lectura intensiva, selección de emergentes y elementos significativos, que en este material sólo focalizaremos en aquellos referidos a las creencias sobre los factores de riesgo y, por lo tanto, a través de las expresiones fundamentalmente de los profesionales de la salud en un contrapunto con los pastores bautistas y los manuales pastoralistas a fin de dar cuenta - aunque mas no sea brevemente - del diálogo entre las explicaciones religiosas y científicas.

\section{Resultados: panorama general del problema}

Las fuentes analizadas revelan los siguientes rasgos más sobresalientes: se analiza el suicidio pero a partir de una frágil conceptualización y una breve expresión escrita, mientras que un $28 \%$ de los trabajos no presenta una definición.

Desde una mirada sociológica hay referencias constantes a obras producidas desde los organismos internacionales (CEPAL, ONU, OMS) y una alta frecuencia de citas de la clásica obra sobre el suicidio de Durkheim (1897) que suele ser presentada como el punto de partida de la discusión sobre la temática. En los trabajos asociados a la salud pública, los factores de riesgo, su identificación y medición ocupan un lugar central.

En este sentido, desde la epidemiología ser joven y varón se presentan como factor de riesgo de conducta suicida. Los jóvenes son vistos como vulnerables, aunque nótese que tanto la edad, el sexo, el origen étnico, son condiciones que no pueden modificarse, de modo que pierden sentido como factores de riesgo, es decir, factores que, en cuanto conocidos, pueden alterarse, prevenirse, modificarse. Incluso, la pobreza estructural cuando se convierte en una condición cotidiana, permanente, es intergeneracional, reduce casi a cero la posibilidad de cambio, de modo que deja de ser riesgo para constituir sufrimiento estructural.

Para la psiquiatría las fuentes más citadas en los artículos provienen de Estados Unidos y Canadá. Hay un énfasis en la descripción de los aspectos neurobiológicos de la conducta suicida (fundamentalmente del sistema serotoninérgico), pero también aparecen con frecuencia cuestiones legales y éticas que hacen a la responsabilidad profesional frente al riesgo de muerte de un paciente.

Desde la psicología se recurre al trabajo con personas sobrevivientes de intentos suicidas. Aunque se hace mención al concepto de autopsia emocional no se han encontrado textos que presenten resultados de ese tipo de análisis. Se trabaja con los casos de intento de suicidio como un modo de acceder a cierta información aunque se reconoce la limitación de su estudio a partir de los datos brindados por sobrevivientes.

Para la filosofía y, en particular, desde el existencialismo, se entiende que los que se suicidan son de ánimo impotente y han sido totalmente vencidos por causas exteriores que repugnan a su naturaleza. El suicidio es paradójico. El suicida quiere terminar con algo que le resulta intolerable y en ese intento termina con su propia existencia, configurando un deseo imposible.

En el caso de los materiales producidos por teólogos - en el campo de la teología práctica - se presenta un recorrido histórico sobre los modos en que la Iglesia ha conceptualizado el suicidio, sobre todo retomando los Concilios en los cuales se fue resolviendo el destino de los cuerpos de los suicidas. Hay cierto consenso en no considerar al suicidio como pecado, sino como el resultado de la enfermedad mental o de la acción demoníaca. Sin embargo en algunos manuales pastoralistas se sigue sosteniendo que el suicidio es un pecado que viola el mandamiento "no matarás", tal como sostenía Santo Tomás de Aquino en el Siglo 13.

En la mayoría de los trabajos aparecen propuestas de intervención desde diferentes actores y en función de los destinatarios del material: profesionales de la salud, psicólogos, pastores o el mismo Estado en términos del diseño de políticas públicas. Se entiende al suicidio como una causa de muerte evitable. La prevención se liga a la identificación de los factores de riesgo antes del intento de suicidio en la intervención individual y a la prevención de la violencia en lo que respecta a la intervención comunitaria.

Hay una gran riqueza en los componentes de las argumentaciones sobre el suicidio juvenil que va desde la clásica apreciación de factores de riesgo hasta la relación con crisis social, desempleo o fracaso escolar. Se ponen en juego tanto los elementos ligados a la enfermedad mental del suicida, su pertenencia a grupos sociales como a problemáticas sociales estructurales. Aparece la construcción cultural que perfila modos de habilitar la violencia en los jóvenes como resultado de la pérdida de valores morales, de la sociabilidad o frente al valor superior de lo material. 
En cuanto a las valoraciones de la conducta suicida también hay una gran variedad de posicionamientos. En un extremo hay quienes lo consideran como el acto de libertad por excelencia: cuando el sujeto decide terminar con su propia vida. En otro extremo podríamos ubicar a quienes ven en el sujeto suicida a un incapaz de decidir libremente, por los condicionamientos que le impone la enfermedad mental. Para algunos es una forma de resistencia, para otros el final de un camino de desesperanza. En las lecturas que lo vinculan al deseo, el suicido es visto como deseo de morir pero también como deseo de una mejor vida. Para los religiosos puede ser visto como un pecado irredimible o como el peor efecto de la acción demoníaca, superando la voluntad del individuo quien juega un papel secundario.

\section{Las creencias sobre los factores de riesgo}

Los profesionales de la salud consideran a la conducta suicida no como un comportamiento exclusivo de la enfermedad mental, sino como una conducta compleja. Se la denomina como proceso suicida o crisis suicida, que tiene una duración promedio pre-establecida de entre 6 a 8 semanas, y se extiende desde la aparición de las primeras ideas de suicidio hasta su resolución (o su repetición) incluyendo o no el pasaje al acto suicida. Planteamientos sobre la neurobiología del suicidio y la concomitante farmacoterapia antisuicida son piezas de esta perspectiva, así como también el trazado de un recorrido que distingue fases: i) pre-suicida; ii) suicida o de pasaje al acto; y iii) post-suicida (de carácter resolutivo y catártico o, por el contrario, como comienzo de una nueva crisis suicida) (LÓPEZ STEINMETZ, 2013). En el marco de una epidemiología de los trastornos mentales, se amplía la comprensión del problema como muerte evitable y prematura y se proyectan las responsabilidades hacia otros actores. Directa o indirectamente se involucra a los profesionales de la salud, pero también a los padres, los maestros, la comunidad, como el propio Estado al que se le reclama intervención.

Por su parte, diversas herramientas de evaluación de riesgos se discuten en la bibliografía, desde Beck et. al. (1979) quien construyera la escala de ideación suicida y luego en Beautrais, (1999), Heriberto (2001), Bongar (2002), entre otros, la mayoría de las cuales cubre múltiples dimensiones y se presentan como listas de control predefinidas para el uso de los evaluadores. Localmente hemos hallado una adaptación realizada con fines de investigación por el equipo de Fernández Liporace y Casullo (2006) de la Universidad de Buenos Aires, del Inventario de Orientaciones Suicidas ISO-30 de King y Kowalchuk (1994), considerando las dimensiones de ideación suicida, desesperanza/desmoralización (o sentimientos negativos), esperanza, relación con los otros y sentimientos positivos, en tanto arreglos a partir de las dimensiones originales: desesperanza, baja autoestima, incapacidad para afrontar emociones, aislamiento social e ideación suicida. Estos instrumentos deben ir acompañados de otro tipo de encuestas que registren datos sociodemográficos.

A pesar de algunas innovaciones, en los materiales relevados hay consenso en afirmar que los factores de riesgo se clasifican en tres grandes grupos: sociodemográficos, clínicos y genéticos/neurobiológicos, según el siguiente esquema:

\section{Cuadro 2: Factores de riesgo en la conducta suicida}

\begin{tabular}{|l|l|}
\hline Clínicos & $\begin{array}{l}\text { Enfermedades que por sí mismas se asocian a trastornos del ánimo - trastornos mentales - } \\
\text { trastornos afectivos - trastornos adaptativos }\end{array}$ \\
\hline Genéticos/neurobiológicos & $\begin{array}{l}\text { Antecedentes familiares - disfunción del sistema serotoninérgico central - transmisión genética } \\
\text { de trastornos psiquiátricos }\end{array}$ \\
\hline Socio-demográficos & Sexo - edad - estado civil - origen étnico - nivel socioeconómico \\
\hline
\end{tabular}

Fuente: Elaboración propia a partir de López Steinmetz (2010), Bongar(2002) y Beck et. al. (1979).

La conducta suicida se analiza a partir de la interacción de al menos dos de estas dimensiones, considerándose a los factores sociales como desencadenantes o precipitantes en concurrencia de aspectos clínicos o genéticos. Cuantos más factores se presenten en un caso aumenta el riesgo de suicidio, lo que explicaría por qué algunas personas se suicidan y otros no ante algún factor similar. De todas formas, la dinámica interactiva de los factores no ha podido determinarse, por lo que el modelo reduce su valor explicativo general, debiendo ponderarse en cada caso particular. Al respecto es ilustrativa la siguiente cita: 


\begin{abstract}
El comportamiento suicida viene determinado por un gran número de causas complejas, tales como la pobreza, el desempleo, la pérdida de seres queridos, una discusión, la ruptura de relaciones y problemas jurídicos o laborales. Los antecedentes familiares de suicidio, así como el abuso de alcohol y estupefacientes, y los maltratos en la infancia, el aislamiento social y determinados trastornos mentales, como la depresión y la esquizofrenia, también tienen gran influencia en numerosos suicidios. Las enfermedades orgánicas y el dolor discapacitante también pueden incrementar el riesgo de suicidio (Comunicado de prensa de la Organización Mundial de la Salud, en ocasión del Día Mundial para la Prevención del Suicidio, 10 de septiembre de 2004).
\end{abstract}

En el caso de los teólogos bautistas, hallamos en los manuales pastoralistas información sobre los factores de riesgo al referirse al suicidio. En El Expositor Bautista ${ }^{2}$ se exponen los predictores de mayor riesgo en adolescentes, entre ellos: intentos o gestos suicidas previos; la existencia de un plan suicida, la disponibilidad de armas de fuego, elementos y medicaciones potencialmente letales en el hogar, un plan o método combinado con el consumo de drogas y alcohol, evidencia de precauciones para no ser rescatado, de poner en orden los asuntos personales, la ideación suicida, el deseo de unirse a un ser amado muerto, la existencia de comunicaciones suicidas orales o escritas, el alejamiento del vínculo con el médico si estuviera en tratamiento y un apoyo, supervisión o juicio parental inadecuados a los pedidos del sufriente. En cuanto a los modos que asumen las relaciones familiares se señalan como factores de riesgo la excesiva rigidez en las expectativas parentales, el abandono físico o emocional, la violencia, antecedentes familiares de enfermedad mental, abuso de alcohol y de drogas. Mientras que respecto a los propios adolescentes se indican problemas interpersonales, bajo control sobre los impulsos, excesiva dependencia emocional de otros, participación en actos de violencia entre compañeros y promiscuidad sexual. En cuanto a los procesos interiores, en un retiro de sanidad interior se enseña que el autorechazo es el principal factor de riesgo de suicidio. Se reconoce la posibilidad de que la persona decida terminar consigo misma por no aceptarse, como resultado de la influencia del rechazo sufrido por otras personas (rechazo prenatal, rechazo de los amigos, de la familia, de la iglesia). Puede derivar en sentimiento de inferioridad, que lleva al perfeccionismo; en predisposición para fracasar, como la sensación de que todo va a salir mal que llena de temor e incapacita para avanzar; temor a las personas y a las nuevas situaciones; en malas ideas sobre sí mismo, pensar mal de uno mismo para castigarse, atormentarse con autocríticas muy duras. Si bien en las entrevistas realizadas a pastores y ministros de sanidad interior no se identifica un planteo de tipo epidemiológico, tales factores funcionan como agravantes en cada caso particular.

los argumentos que enuncian la prevención del suicidio juvenil son discursos basados en un recurso retórico como el ejemplo. Ello reduce las posibilidades de intervención social en la medida que sostienen la idealización de la juventud y su contracara: la negativización de sus problemas o crisis expresadas en la ideación de muerte.

En otro orden, hallamos que tanto los discursos científicos como religiosos analizados apelan recurrentemente a un recurso argumentativo basado en el ejemplo, es decir, en una definición del debe ser del joven, de la familia, de la escuela, como del propio Estado con miras a prevenir el suicidio. Estas formas narrativas procuran delinear lo ejemplar, el patrón de normalidad, el joven ideal, en relación con las conductas que tienen que llevar a cabo una persona o grupo. Distinguen lo que es legítimo de lo que no lo es, y establecen lo que debe y lo que no debe hacerse o creerse. Cuando los autores ofrecen estas descripciones no contraponen ninguna forma de prueba científica que fundamente que así debe ser. Solo se lo presenta con una contundencia rotunda: la familia debe ser estable, la sociedad inclusiva, la escuela debe educar para prevenir y con capacitación y disposición lo logrará, los docentes deben ser comprensivos, comunicativos y flexibles, el joven debe proyectarse al futuro definiendo un proyecto vital, debe ser consciente de ello así puede actuar con relativa racionalidad, estar integrado al mercado laboral o productivo, o en vías de hacerlo, mientras que el Estado debe intervenir más.

En suma, el análisis de los factores de riesgo está presente tanto en la perspectiva científica como religiosa en la medida que ello posibilita la prevención, aunque sostenido en los patrones de la epidemiológica clásica, lo cual habla de una creencia fuertemente acendrada en el contexto en el que circula. Asimismo, el estudio de factores aporta esencialmente a la prevención primaria del suicidio, por cuanto la prevención secundaria focaliza en la atención psicológica al joven que ha intentado suicidarse, mediante acciones de protección que algunos llaman clínica del desamparo, mientras la prevención terciaria se concentra en la 
atención a los sobrevivientes de un suicidio atendiendo a familiares, amigos, convivientes que podrían estar en riesgo no sólo por la ocurrencia del efecto imitación sino por el contexto de elaboración del duelo y posibles derivaciones en procesos depresivos.

\section{Análisis}

Como anticipamos, los argumentos que enuncian la prevención del suicidio juvenil, son discursos basados en un recurso retórico como el ejemplo. Ello reduce las posibilidades de intervención social en la medida que sostienen la idealización de la juventud y su contracara: la negativización de sus problemas o crisis expresadas en la ideación de muerte. Dicha idealización (también de la familia y de la sociedad) se refleja en unas intervenciones preventivas fundadas en la identificación de factores de riesgo. Sin embargo, estos no se problematizan, tampoco se amplían en indicadores más certeros para su detección, en especial en lo atinente a los factores sociales como a los factores protectores. Se mantienen las referencias a atributos humanos que hacen al genotipo y fenotipo los que no se pueden modificar mediante la acción planificada, prescindiendo de las construcciones culturales y de poder que generan tales atributos las que si en cambio, podrían ser objeto de intervención. Pareciera que al enunciar la categoría factores de riesgo o factores protectores no hay nada más que explicar, o más bien, ya estaría todo dicho. Los científicos creen efectivamente en la capacidad explicativa de estos conceptos y en su aplicación lineal. Incluyen definiciones tácitas, no se los cuestiona, no se los analiza, se los emplea sin más aun cuando operativamente resulte insuficiente para elaborar una propuesta de acción.

Respecto de los factores de riesgo que conllevan en su concepción una idealización de las condiciones de vida, pero también un reduccionismo y un vaciamiento de contenido histórico, deben reelaborarse a partir de las realidades locales, con insumos que los propios servicios socios-sanitarios están en condiciones de proveer. $\mathrm{Al}$ respecto podemos ampliar y precisar el esquema básico de factores de riesgo a modo de elaboración inicial, considerando para ello, como señala Breilh (2003), una lógica general de la reproducción social, estilos de vida personales y fenómenos geno-fenotípicos:

\section{Cuadro 3: Reelaboración de la matriz de factores de riesgo en la conducta suicida}

\begin{tabular}{|c|c|}
\hline $\begin{array}{l}\text { Psicosociales de base } \\
\text { clínica, con o sin } \\
\text { origen genético o } \\
\text { neurobiológico }\end{array}$ & $\begin{array}{l}\text {-condiciones de stress } \\
\text {-rasgos de personalidad } \\
\text {-formas de afrontamiento de problemas } \\
\text {-problemas de manejo en la vida cotidiana } \\
\text {-impulsividad, ira, enojo } \\
\text {-abuso de sustancias } \\
\text {-antecedentes de abuso sexual } \\
\text {-rasgos psicóticos } \\
\text {-depresión } \\
\text {-salud y problemas médicos (severidad, naturaleza) }\end{array}$ \\
\hline $\begin{array}{l}\text { Apoyo social y } \\
\text { recursos (cognitivos, } \\
\text { emocionales, } \\
\text { materiales) }\end{array}$ & $\begin{array}{l}\text {-familia de origen, memoria familiar } \\
\text {-existencia de roles de apoyo, ayuda y cuidado en función de las necesidades del ciclo vital } \\
\text {-existencia de redes de apoyo social, participación en grupos e instituciones, integración comunitaria } \\
\text {-recursos materiales y simbólicos (carencia de bienes básicos, seguridad física y económica) } \\
\text {-vivencias y experiencias del hábitat, de la escolaridad y el trabajo, de exclusión, discriminación y } \\
\text { disciplinamiento, y del proceso de salud-enfermedad-atención-cuidado }\end{array}$ \\
\hline Proceso suicida & $\begin{array}{l}\text {-ideación y pensamientos suicidas } \\
\text {-historia de intentos (número de intentos, letalidad) } \\
\text {-plan suicida (detalles, disponibilidad de medios, tiempo, letalidad del método, oportunidad de intervención } \\
\text { externa, arreglos finales) }\end{array}$ \\
\hline
\end{tabular}

Fuente: Elaboración propia en base a Barrón (2015), Ponce de León y Krmpotic (2012), Cheung, Wang y Leung (2011) y Breilh (2003).

Asimismo, para mejorar su incidencia en la acción, la ponderación de factores de riesgo debería dar lugar a escalas, cuyos niveles (bajo, medio o alto) estén determinados por: la existencia del factor (el plan 
suicida, el abuso de sustancias, la historia de abuso sexual); la frecuencia (de la ideación suicida, la historia de intentos); y la severidad (del stress, la impulsividad, falta de apoyo social), integrando distintas fuentes y tipos de información, sea brindada por los sufrientes, pares, familias, o a partir de la evaluación de agentes de los servicios sociales y de salud (trabajadores sociales, psicólogos, médicos etc.) junto a los datos estadísticos que procesan las instituciones que atienden problemas sociales y sanitarios, desde un nivel macro-institucional como microsocial.

Finalmente, la prevención del suicidio en base a factores de riesgo podría pasar del discurso predominante a la acción en la medida que se cumplan determinadas condiciones: un registro sistemático de casos que permitan actualizar y ajustar el sistema de factores de riesgo en función de las realidades locales; un sistema de información que mantenga en-línea esos registros, de modo de brindar apoyo a la decisión rápida en la evaluación de riesgos de suicidio; un sistema de alerta interinstitucional geo-referenciado; la reutilización del conocimiento organizacional, el conocimiento basado en la sistematización de casos debe complementar la experiencia y habilidades de los profesionales intervinientes, en la medida que la calidad de un servicio depende de ellas. Los intercambios cara a cara entre los trabajadores sociales y otros profesionales, continúan siendo herramientas valiosas por su carácter altamente contextual y eficaz, en el proceso de generación de conocimientos basados en la práctica; el estudio de casos como el intercambio grupal en la conformación de un inventario básico de factores riesgo, puede ampliar su alcance a otros ámbitos cuyas decisiones también se basan en modelos de riesgo, como en los servicios sociales de protección a la infancia o a las familias, en particular cuando comparten similar población destinataria, ámbito geográfico o contextos multiproblemáticos; respecto del papel de los agentes profesionales, y atendiendo en particular al Trabajador Social, éste debe agudizar su mirada, mejorar los instrumentos de captación de la realidad y compartir el estado actual de los conocimientos en la materia con el equipo multiprofesional. Al respecto, deberá procurar comprender en profundidad no sólo aquello que atañe a la dimensión social sino ser capaz de articular con aquella los factores psico-sociales y del proceso suicida. Un sistema de alerta temprana no resiste un trabajo profesional aislado, como tampoco alejado de la comunidad en que el servicio se inserta. La prevención involucra el componente educativo que será reelaborado en términos del sentido común local, para que la red preventiva efectivamente funcione.

\section{Conclusiones}

¿Cuáles son las condiciones necesarias para pasar de la enunciación a la acción en la mortalidad juvenil?, pues retomando la reflexión de Krmpotic (2005) ¿ será acaso que en los propios argumentos disciplinarios se hallan los límites para la acción?

Si bien estas muertes jóvenes producen una ruptura de sentido no llegan a movilizar suficientemente decisiones políticas. La intervención en adolescentes y jóvenes - comparativamente con la de niños y los ancianos - presenta un carácter marginal que si bien afecta a un grupo social numeroso, se desenvuelve con acciones puntuales, fragmentadas y escasas, y con un énfasis llamativo en la inserción laboral como llave para resolución de todos los conflictos. Como otras tantas muertes en la trayectoria del país, pensar en la historia del suicidio como en una historia social del dolor es una metáfora elocuente. El sufrimiento que se genera frente a la ocurrencia del suicidio es social. Las pérdidas que se producen afectan a la sociedad en su conjunto. Su medición a través del índice de AVPP (años de vida potencialmente perdidos) dice del problema en algunos aspectos, pero tiene implicancias cualitativas mucho más complejas de ponderar en frecuencia e intensidad.

Los estudios sobre factores de riesgo dan cuenta de algunos aspectos del fenómeno, por lo que se justifica el esfuerzo en desarrollar herramientas de evaluación realistas, dinámicas, que amplíen fundamentalmente los convencionales factores sociales tan pobremente descriptos pero tan determinantes dada la condición aceptada de desencadenantes. No se trata de reificar factores, muy por el contrario, de superar la ambigüedad interpretativa que presentan en el caso de la prevención del suicidio. El Trabajo Social en particular, tienen en este sentido mucho para aportar, con profesionales preparados para el abordaje de la complejidad en la interacciones entre los sujetos, lo institucional y lo comunitario, así como para comprender las intersecciones entre los modos de vida, la episteme - es decir las reglas y presuposiciones que rigen los discursos científicos, políticos, religiosos - y la cultura.

\section{Referencias}

BARRÓN, E. V. Creencias en torno al suicidio juvenil. Intersecciones argumentativas entre religión y abordaje científico. Tesis para obtener el grado de Doctor en Ciencias Sociales. Buenos Aires, Facultad de Ciencias Sociales, Universidad de Buenos Aires, 2015. 
BEAUTRAIS, A. L. Risk Factors for Suicide and Attempted Suicide Among Young People. Australian and New Zealand Journal of Psychiatry. The Royal Australian and New Zealand College of Psychiatrists: Sage, v. 34, p. 420-436, 1999.

BECK, A.T.; KOVACS M.; WEISSMAN A. Assessment of Suicidal Intention: The Scale for Suicide Ideation. Journal of Consulting and Clinical Psychology. Washington, DC: American Psychological Association, v. 47, n. 2, p. 343-352, 1979.

BONGAR, B. The Suicidal Patient: Clinical and Legal Standards of Care. Washington, DC: American Psychological Association, 2002. BREILH, J. Epidemiología crítica. Ciencia emancipadora e interculturalidad. Buenos Aires: Lugar Editorial, 2003

CHEUNG, C. F.; WANG, W. M.; LEUNG, Z. C. A pilot study on a knowledge-based case library to support suicide risk assessment. International Social Work. International Association of Schools of Social Work: Sage, v. 56, n. 2, p. 208-227, 2011.

FERNÁNDEZ LIPORACE, M. y CASULLO, M. M. Validación factorial de una escala para evaluar riesgo suicida. Revista Iberoamericana de Diagnóstico y Evaluación Psicológica. Buenos Aires: Asociación Iberoamericana de Diagnóstico y Evaluación Psicológica (AIDEP), v. 21, n. 1, p. 9-22, 2006.

HERIBERTO, G. S. Risk Factor Model for Suicide Assessment and Intervention. Professional Psychology: Research and Practice. Washington, DC: A.P.A, v. 32, n. 4, p. 351-358, 2001.

KING, J. D. y KOWALCHUK, B. Adolescent Inventory of Suicide Orientation - 30. Minneapolis: National Computer Systems, 1994. KRMPOTIC, C. (coord.) Trabajo duro, trabajo sucio. La inserción laboral de jóvenes residentes en barrios críticos. Buenos Aires: Espacio Editorial, 2005.

LOPEZ STEINMETZ, L. C. Invisibilidad de los riesgos e inequidad social. Un análisis psicológico en casos de intento de suicidio (Jujuy, 2007-2008). In: KRMPOTIC, C.; MITJAVILA, M.; SAIZAR, M. M. (eds.) (Sub) Culturas profesionales. Poder y prácticas en salud. Buenos Aires: Miño y Dávila, 2013, p. 171-188.

PIÑUEL RAIGADA, J. y J. A. GAITÁN MOYA. Metodología general. Conocimiento científico e investigación en la comunicación social. Madrid: Síntesis, 1995.

PONCE DE LEÓN, A.; KRMPOTIC, C. Trabajo Social Forense. Balance y perspectivas. Buenos Aires: Espacio Editorial, 2012.

ORGANIZACIÓN MUNDIAL DE LA SALUD. El suicidio, un problema de salud pública enorme y sin embargo prevenible. Comunicado de prensa, 8 de septiembre de 2004.

ROBSON, C. Real World Research. A Resource for Social Scientists and Practitioner-Researchers. Oxford: Blackwell, 2002.

SERFATY, E. Suicidio en adolescentes: aspectos epidemiológicos y genéticos. Revista Argentina de Psiquiatría Biológica. Buenos Aires, v. 12, n. 92, p. 5-6, 2005.

SCHÜTZ, A. Fenomenología del mundo social. Buenos Aires: Paidos, 1972.

YUNES, J.; ZUBAREW, T. Mortalidad por causas violentas en adolescentes y jóvenes: un desafío para la región de las Américas. Revista Brasileira de Epidemiolología. São Paulo: Associação Brasileira de Saúde Coletiva, v. 2, n. 3, p. 102-171, 1999.

\section{Notas}

1 Este artículo exhibe parcialmente los resultados de un estudio mayor en el marco de una tesis doctoral, en el que se desarrolló además una etnografía de casos de intento suicida como entrevistas en profundidad a protagonistas del proceso de cura.

2 Se trata de una publicación de periodicidad mensual de la Confederación Bautista Argentina.

\section{Elsa Viviana Barrón}

vivibarron@hotmail.com

Doutora em Ciências Sociais (UBA)

Professora nas Universidades Nacionais de Buenos Aires e de Matanza.

\section{Claudia Sandra Krmpotic}

csk@fibertel.com.ar

Doutora em Serviço Social (PUC/SP)

Professora nas Universidades Nacionais de Buenos Aires e Matanza.

Investigador no Centro Argentino de Etnología Americana - CONICET

\section{UBA}

Faculdade de Ciencias Sociais

Santiago del Estero 1029

Cidade Autónoma de Buenos Aires, Argentina

CP. 1075 


\section{CAEA-CONICET}

Av. de Mayo $1437,1^{\circ} \mathrm{A}$

Cidade Autónoma de Buenos Aires, Argentina

CP. 1085 